\title{
Adaptação Transcultural Brasil-Portugal da Escala de Satisfação com o Suporte Social para Estudantes do Ensino Superior
}

\author{
Brazil-Portugal Transcultural Adaptation of the Social Support Satisfaction \\ Scale for College Students
}

\author{
João P. Marôco*, a, Juliana Alvares Duarte Bonini Campos ${ }^{b}$, Maria da Graça Vinagre ${ }^{c}$ \\ \& José Luís Pais-Ribeiro ${ }^{d}$ \\ ${ }^{a}$ Instituto Superior de Psicologia Aplicada, Lisboa, Distrito de Lisboa, Portugal, \\ ${ }^{b}$ Universidade Estadual Paulista, Araraquara, São Paulo, Brasil, \\ ${ }^{c}$ Escola Superior de Enfermagem de Lisboa, Lisboa, Distrito de Lisboa, Portugal \\ \& ${ }^{d}$ Universidade do Porto, Porto, Distrito do Porto, Portugal
}

\begin{abstract}
Resumo
Realizou-se adaptação transcultural da Escala de Satisfação com o Suporte Social (ESSS) para a língua portuguesa. As qualidades psicométricas foram avaliadas numa amostra de 1.023 estudantes do ensino superior do Brasil e de Portugal. A partir dos resultados obtidos propõe-se uma versão modificada da ESSS com 12 itens que avaliam 4 dimensões. A versão modificada revelou adequada confiabilidade, validade fatorial, validade concorrente, divergente e discriminante com exceção dessa última para Satisfação com as Amizades e a Intimidade. A validade convergente esteve no limite do aceitável. Observou-se invariância dos pesos fatoriais entre Brasil e Portugal, permitindo sua utilização para a avaliação da Satisfação com o Suporte Social em estudantes do ensino superior de ambos os países. Palavras-chave: Suporte social, estudantes, escalas psicométricas.
\end{abstract}

\begin{abstract}
The Social Support Satisfaction Scale (SSSS) was submitted to an adaptation to Portuguese language. Psychometric qualities of the SSSS were assessed in a sample of 1,023 university and college students from Brazil and Portugal. From the results obtained, we propose a modified version of the SSSS with 12 items that assess four dimensions (Satisfaction with Friendship, Intimacy, Satisfaction with Family and Social Activities). The modified version of the SSSS demonstrated good reliability; factorial validity; and concurrent, divergent and discriminant validity. Convergent valid is in the limit of what is acceptable. There was invariance of the factorial weights in the Portuguese and Brazilian samples, allowing its use for assessing "Social Support Satisfaction" in higher education students in both countries.

Keywords: Social support, students, psychometric scales.
\end{abstract}

O Suporte Social (SS) tem sido fruto de múltiplas conceptualizações, desde os aspectos mais objectivos de ordem social como o número de amigos, frequência e intensidade de contactos, existência de redes sociais, aos aspectos subjetivos de ordem cognitiva como a perceção do individuo de ser apoiado, e de ordem avaliativa no que se refere à "adequação do" e "satisfação com" o apoio. Em geral, pode definir-se SS como "a existência ou disponibilidade de pessoas em quem podemos confiar, que nos deixam entender que se preocupam conosco, que nos valorizam e que gostam de nós" (Sarason, Levine, Basham, \& Sarason, 1983). Entre a diversidade de conceitos salientamos o que

\footnotetext{
*Endereço para correspondência: Instituto Superior de Psicologia Aplicada, Rua Jardim do Tabaco, 34, Lisboa, Portugal 1149-041. E-mail: jpmaroco@ispa.pt, jucampos@foar.unesp.br, gvinagre@esel.pt e jlpr@psi.up.pt
}

se refere ao suporte social como o conjunto de informação que leva o indivíduo a acreditar que é apoiado e amado, que é estimado e valorizado, que pertence a uma rede de comunicação e de obrigações mútuas (Cobb, 1976). Autores como Cramer, Henderson e Scott (1997) fazem a distinção entre SS percebido e SS recebido. O primeiro refere-se à perceção que o indivíduo tem do suporte social disponível quando dele necessita, o segundo diz respeito aquele que é efetivamente recebido de alguém. Em situações em que as pessoas têm limitações funcionais o suporte social recebido ou tangível é mais utilizado. Exemplo de um instrumento desse tipo é a escala de suporte social do MOS (MOS Social Support Survey, de Sherbourne \& Stewart, 1991), com versão em português europeu de Pais-Ribeiro e Ponte (2009) e em português do Brasil de Chor, Griep, Lopes, e Faerstein (2001). Suporte social e apoio social são utilizados como sinónimos do termo que em inglês seria "social support". No Brasil é mais frequente 
utilizar o termo apoio (Gonçalves, Pawlowski, Bandeira, \& Piccinini, 2011).

Atualmente, o suporte social ou apoio social é considerado protetor frente às adversidades que podem ocorrer no ambiente escolar (Demaray \& Malecki, 2006; Rothon, Head, Klineberg, \& Stansfeld, 2011) ou no contexto de trabalho (Taylor, 2008). De acordo com a literatura, o suporte social está associado à menor mortalidade e morbilidade (Uchino, 2006) podendo fornecer benefícios substanciais para a saúde e o bem estar dos indivíduos (Gecková, van Dijk, Stewart, Groothoff, \& Post, 2003; Taylor et al., 2010).

Os estudos de investigação sobre o suporte social são consensuais quanto à importância desta variável (Berkman \& Glass, 2000; Callaghan \& Morrissey, 1993; Cohen \& Lemay, 2007; Diong et al., 2005) relatando que a disponibilidade de alguém que forneça ajuda ou apoio emocional constitui um fator protetor frente às situações estressantes e, em particular, diante de consequências negativas de doenças graves (Sherbourne \& Stewart, 1991). O funcionamento social é um conceito amplo e genérico que pode incluir todo o comportamento humano no papel e contexto social (Sherbourne, 1992). Singer e Lord (1984) explicam que o SS protege das perturbações induzidas pelo estresse e que sua ausência pode ser um fator estressor.

O suporte social é pois um construto complexo e multidimensional que se relaciona com a saúde e bem-estar do indivíduo, contribuindo para a compreensão de como as diferentes relações têm influência positiva ou negativa na forma como o indivíduo lida com as adversidades com que se confronta no decurso do seu desenvolvimento. Assim, mensurar o suporte social pode ser uma estratégia interessante para deteção de situações de desenvolvimento de comportamentos de risco. Na década de 90 foi desenvolvida por Pais-Ribeiro (1999) uma escala para avaliar o SS percebido, a Escala de Satisfação com o Suporte Social (ESSS). Esta escala avalia a satisfação que o indivíduo sente em relação ao apoio social que pensa ter disponível. Inclui 15 itens que se distribuem por quatro dimensões: "Satisfação com os amigos", "Intimidade", "Satisfação com a família", e "Atividades Sociais". Em Portugal, essa escala tem sido utilizada em diferentes populações, com várias idades, normativas ou que apresentem alguma patologia como neoplasias (Santos, Pais-Ribeiro, \& Lopes, 2003), acidente vascular cerebral e doenças cardiovasculares (Coelho \& Pais-Ribeiro, 2000; Ferreira, Pais-Ribeiro, \& Guerreiro, 2004), diabetes (Silva, Pais Ribeiro, Cardoso, \& Ramos, 2003) ou doença mental (Pais-Ribeiro \& Guterres, 2001) e tem-se mostrado útil nestas diferentes populações. Contudo, tanto quanto sabemos, a ESSS não tem sido aplicada em outros países de língua portuguesa, nem conhecemos a existência de investigações que tenham recorrido à ESSS, avaliando as suas qualidades psicométricas fora de Portugal ou tentado a sua adaptação transcultural. A ESSS foi utilizada no Brasil com gestantes por Baptista, Baptista, e Torres (2006), apenas com ajustamento da grafia para o português do Brasil.
A adaptação transcultural é um processo fundamental quando um instrumento desenvolvido numa determinada população pretende ser aplicado em outra população diferente. He e Van de Vijver (2012) explicam que "a aplicação de um novo instrumento, num grupo cultural novo, envolve mais do que a simples produção de texto noutra língua, administrar o instrumento traduzido, e comparar os resultados". Bradley (1994) afirma que uma nova tradução necessita ser submetida aos mesmos procedimentos que é suposto a versão original ter sofrido, de tal forma que a avaliação da validade de conteúdo e de construto se sobreponha ao processo de tradução (De Raad, 1998; He \& Van de Vijver, 2012; Herdman, Fox-Rushby, \& Badia, 1998).

He e Van de Vijver (2012) têm defendido que os estudos transculturais devem ser apresentados com todas as etapas de avaliação psicométrica o que requer uma amostra substancial, um desenho de investigação adequado e a demonstração de que o construto, método e viés do item não diferem entre as versões. Pelo fato de entendermos que todos estes aspectos são relevantes, no presente estudo, cuidamos de identificar o viés e as equivalências da ESSS quando aplicada em Portugal e no Brasil, dado tratar-se de duas culturas diferentes. Portugal e Brasil são países cuja língua nativa é o português e desde 2009, quando do estabelecimento do acordo ortográfico, passaram a ter sua escrita uniformizada. No entanto, diante das peculiaridades culturais dos dois países, por vezes, são utilizadas palavras iguais para expressar objetos diferentes ou palavras que não são utilizadas no outro país.

Do mesmo modo, diferentes culturas utilizam o suporte social de forma diferente (Kim, Sherman, \& Taylor, 2008) e, portanto, deve-se verificar se os itens, componentes do instrumento, são entendidos do mesmo modo (equivalência lexical) e se apresentam o mesmo significado (equivalência cultural).

Assim, realizou-se este estudo, de adaptação transcultural da ESSS com os objetivos de (a) apresentar uma escala na língua portuguesa, para estudantes do ensino superior, que possa ser utilizada tanto no Brasil quanto em Portugal; (b) que tenha uma estrutura semelhante para os dois países; (c) uniformizar o número de itens por dimensão; (d) melhorar a qualidade do ajustamento do modelo fatorial sem comprometer a estrutura cognitiva; (e) avaliar as qualidades psicométricas da ESSS, nas suas facetas da sensibilidade, validade de construto (fatorial, convergente e discriminante), validade de critério (concorrente e divergente) e confiabilidade da medida, incluindo a invariância do modelo fatorial em amostras de estudantes do ensino superior do Brasil e Portugal,.

\section{Método}

\section{Participantes}

Participaram deste estudo 1.023 estudantes do ensino superior dos quais $69 \%$ eram do sexo feminino. Desses, 532 eram brasileiros e 491 portugueses. A média de idade dos participantes foi 22,7 anos $(D P=5,5)$. Com relação à 
Marôco, J. P., Campos, J. A. D. B., Vinagre, M. G. \& Pais-Ribeiro, J. L. (2014). Adaptação Transcultural Brasil-Portugal da Escala de Satisfação com o Suporte Social para Estudantes do Ensino Superior.

área do curso de graduação, $66 \%$ dos participantes pertenciam às ciências da saúde, $16 \%$ eram das ciências exatas, $15 \%$ da área das ciências sociais e humanas e apenas $3 \%$ relataram ser da área de ciências biológicas. Sessenta por cento dos alunos frequentava uma instituição de ensino superior público. As informações demográficas por país de origem são apresentadas na Tabela 1.

Tabela 1

Caracterização dos Participantes do Estudo de Adaptação da ESSS, Organizados por País

\begin{tabular}{lccc}
\hline \multirow{2}{*}{ Indicador } & \multicolumn{2}{c}{ País } & \multirow{2}{*}{ Total } \\
\cline { 2 - 3 } & Brasil & Portugal & \\
\hline$n$ & 532 & 491 & 1.023 \\
Idade $[M(D P)]$ & $22,4(5,6)$ & $23,0(5,3)$ & $22,7(5,5)$ \\
Sexo - \% & & & \\
Masculino & 40 & 18 & 31 \\
Feminino & 60 & 82 & 69 \\
Escola - \% & & & \\
Privada & 70 & 4 & 40 \\
Publica & 30 & 96 & 60 \\
Área do curso - \% & & & \\
Ciências Biológicas & 4 & - & 3 \\
Ciências da Saúde & 40 & 95 & 66 \\
Ciências Exatas & 30 & 1 & 16 \\
Ciências Humanas & 26 & 4 & 15 \\
e Sociais & & & \\
\hline
\end{tabular}

\section{Instrumento}

Como instrumento de medida utilizou-se a escala de Satisfação com o Suporte Social (ESSS) proposta por Pais-Ribeiro (1999) que é constituída por 15 afirmações relativas à perceção de suporte e apoio que os respondentes recebem dos amigos, família e comunidade que encontram-se distribuídas em quatro fatores (Tabela 2). A escala é de autopreenchimento com um formato de resposta de Likert com 5 pontos $(5$ - Concordo totalmente, 4 - Concordo na maior parte, 3 - Não concordo nem discordo, 2 - Discordo na maior parte e 1- Discordo totalmente). No estudo original (Pais-Ribeiro, 1999) a escala foi aplicada a uma amostra portuguesa de estudantes de 15 a 30 anos de idade e na análise fatorial exploratória foram retidos 4 fatores que explicaram $63 \%$ da variância total. A consistência interna foi adequada (Escala: $\alpha=0,85$; Fatores: $\alpha=0,64-0,83$ ).

$\mathrm{Na}$ versão original os itens foram cotados de forma inversa. Nesta aplicação da ESSS a codificação numérica foi invertida para que a um maior valor de código numérico correspondesse uma maior concordância. É de notar que os itens SS1, SS6, SS9, SS13, SS14 e SS15 estão formulados de forma negativa de modo que, antes da realização das análises, as pontuações destes itens foram revertidas. Os itens do questionário foram revisados e adaptados segundo o acordo ortográfico que unifica a escrita do português em países em que esta é a língua oficial. Esse acordo entrou em vigor em 2009. Após revisão dos itens, os mesmos foram checados quanto às equivalências semânticas, idiomáticas e culturais por dois profissionais da área de Psicologia sendo um português e um brasileiro.

Para possibilitar a avaliação da validade concorrente da ESSS utilizou-se também a versão em português da Escala de Percepção de Suporte Social (EPSS) proposta por Siqueira (2008) composta por 29 itens, distribuídos em três fatores (Suporte emocional, Suporte instrumental e Suporte de Informação). Trata-se de escala do tipo Likert de 4 pontos (1: Nunca, 4: Sempre). Nesse estudo, optou-se por adicionar um ponto central à escala de modo que a mesma passou a variar de 1 (Nunca) a 5 (Sempre). Para avaliar a validade divergente da ESSS utilizou-se a versão em português do Inventário de Burnout de Maslach (MBI-SS), apresentada por Campos, Zucoloto, Bonafé, Jordani e Maroco (2012). A MBI-SS é composta por 15 itens subdivididos em 3 fatores (Exaustão, Descrença, Eficácia Profissional). A escolha da MBI-SS para avalizar a validade divergente decorre do fato de o Suporte Social poder ser um atenuador da síndrome de Burnout, sendo expectável correlações negativas entre as dimensões dos dois construtos.

\section{Procedimento}

O procedimento de aplicação dos questionários foi distinto no Brasil e em Portugal. No Brasil, foi elaborado um site, utilizando o googledocs, para abrigar o questionário demográfico e as escalas ESSS, EPSS e MBI-SS. Os questionários ficaram disponíveis on-line para preenchimento durante 12 meses. Cada página da internet abrigou um inventário de modo que o respondente poderia visualizar todos os itens de cada instrumento simultaneamente. Foram permitidas não resposta ao item e o participante poderia retornar para verificar e/ou corrigir a resposta dada a cada inventário antes de sua submissão. A divulgação do endereço eletrônico foi realizada junto à direção das escolas de ensino superior cadastradas junto ao Ministério da Educação e Cultura e também junto às redes sociais. Em Portugal, os instrumentos foram disponibilizados em papel. A participação dos estudantes foi voluntária e os mesmos preencheram os questionários, em sala de aula, de forma individual. A realização dessa pesquisa foi autorizada pelas direções de instituições de ensino superior. É de referir que relativamente às diferenças de formato de apresentação dos instrumentos, os dados publicados em estudos recentes permitem concluir que, na avaliação realizada com os instrumentos em papel e no ecrã do computador, as duas formas de recolha de dados são equivalentes (Campos et al., 2011; Gwaltney, Shields, \& Shiffman, 2008).

Tanto os estudantes brasileiros quanto portugueses, foram informados, por escrito, que a sua participação era voluntária, anônima e não remunerada. Os objetivos da 
Psicologia: Reflexão e Crítica, 27(2), 247-256.

Tabela 2

Escala de Satisfação com o Suporte Social - ESSS (Pais-Ribeiro, 1999)

Item $^{\#}$

Fator

SS1r. Os meus amigos não me procuram tantas vezes quantas eu gostaria

Satisfação com

SS2. Estou satisfeito com a quantidade de amigos que tenho

Amizades (SA)

SS3. Estou satisfeito com a quantidade de tempo que passo com os meus amigos

SS4. Estou satisfeito com as atividades e coisas que faço com meu grupo de amigos

SS5. Estou satisfeito com o tipo de amigos que tenho

SS6r. Por vezes sinto-me só no mundo e sem apoio

Intimidade (IN)

SS7. Quando preciso desabafar com alguém encontro facilmente amigos com quem o fazer

SS8. Mesmo nas situações mais embaraçosas, se precisar de apoio de emergência tenho várias pessoas a quem posso recorrer

SS9r. Às vezes sinto falta de alguém verdadeiramente íntimo que me compreenda e com quem possa desabafar sobre coisas íntimas

SS10. Estou satisfeito com a forma como me relaciono com a minha família

Satisfação com

SS11. Estou satisfeito com a quantidade de tempo que passo com a minha família

a família (SF)

SS12. Estou satisfeito com o que faço em conjunto com a minha família

SS13r. Não saio com amigos tantas vezes quanto eu gostaria

Atividades Sociais

SS14r. Sinto falta de atividades sociais que me satisfaçam

(AS)

SS15r. Gostaria de participar mais em atividades de organização (p. ex. clubes desportivos, escoteiros, partidos políticos, etc)

Nota. Os itens com "r" no final devem ser revertidos.

pesquisa foram claramente explicitados e os participantes foram informados que o estudo era de natureza estritamente acadêmica e não tinha qualquer objetivo de diagnóstico e/ ou intervenção. Os aspetos éticos da pesquisa com seres humanos foram avaliados em Portugal pela comissão científica da Unidade de Investigação em Psicologia e Saúde (UIPES) e, no Brasil, pelo Comitê de Ética em Pesquisa em Seres Humanos da Faculdade de Ciências Farmacêuticas da UNESP-Araraquara (protocolo CEP/ FCF/Car n ${ }^{\circ}$ 1/2011).

\section{Avaliação das Qualidades Psicométricas}

A avaliação das qualidades psicométricas dos dados foi realizada estimando-se a sensibilidade psicométrica, a validade e a confiabilidade. Todas as análises foram efetuadas no programa IBM SPSS Statistics (v. 20, SPSS An IBM Company, Chicago, IL) e IBM SPSS AMOS (v. 20, SPSS An IBM Company, Chicago, IL).

\section{Sensibilidade Psicométrica}

Considerou-se que os itens que apresentavam distribuição de valores entre o mínimo e o máximo da escala, com valores absolutos de assimetria e curtose inferiores a 3 e 7, respectivamente, (Kline, 2004; Maroco, 2010) apresentaram sensibilidade psicométrica.

\section{Validade Relacionada com o Construto}

A validade relacionada com o construto foi avaliada por meio da validade fatorial, convergente e discriminante. A validade fatorial foi testada com Análise Fatorial Confirmatória realizada, com estimação de máxima verosimilhança. Considerou-se que o modelo fatorial proposto tinha validade quando todos os itens apresentaram pesos fatoriais superiores a 0,4 e o modelo de 4 fatores apresentou um bom ajuste à estrutura de variância-covariância avaliado pelos índices de qualidade de ajustamento. Os índices e respectivos valores de referência utilizados para avaliar a qualidade do ajustamento foram: Razão da Estatística do Qui-quadrado pelos graus de liberdade $\left(\chi^{2} / \mathrm{gl}\right)$ inferior a 3,0, Goodness of Fit Index (GFI), Comparative Fit Index (CFI) e Tucker-Lewis Index (TLI) superiores a 0,9 e Root Mean Square Error of Aproximation (RMSEA) inferior a 0,05 , com limite superior do intervalo de confiança a $90 \%$ inferior a 0,10 (Kline, 2004; Maroco, 2010). 
Marôco, J. P., Campos, J. A. D. B., Vinagre, M. G. \& Pais-Ribeiro, J. L. (2014). Adaptação Transcultural Brasil-Portugal da Escala de Satisfação com o Suporte Social para Estudantes do Ensino Superior.

A validade convergente foi estimada pela Variância Extraída Média (VEM) e foi considerada adequada se $\mathrm{VEM} \geq 0,50$ (Fornell \& Larcker, 1981; Maroco, 2010). A validade discriminante foi avaliada comparando a VEM para cada fator com o quadrado da correlação de Pearson entre os fatores. Adotou-se a proposta de Fornell e Larcker (1981) de que existe validade discriminante quando a VEM de cada fator é superior ao quadrado da correlação entre fatores.

Invariância do Modelo Fatorial em Portugal e no Brasil

A invariância do modelo fatorial e a estabilidade transcultural Brasil-Portugal foram avaliadas por meio de análise de equações estruturais multigrupos. $O$ teste de invariância foi realizado impondo-se restrições de igualdade aos pesos fatoriais das duas amostras sendo a estatística de teste a da diferença dos $\chi^{2}\left(\Delta \chi^{2}\right)$ do modelo com pesos fatoriais fixos e do modelo com pesos iguais. Quando a hipótese da invariância dos pesos fatoriais foi aceita realizou-se a análise da invariância das covariâncias entre fatores e da invariância dos fatores específicos (resíduos; Maroco, 2010).

\section{Validade Relacionada com um Critério}

A validade relacionada com um critério foi avaliada nas suas facetas de validade concorrente e validade divergente. A validade concorrente foi analisada por correlação de Pearson com as dimensões da Escala de Percepção do Suporte Social (EPSS) adaptada por Siqueira (2008) para a língua portuguesa. A validade divergente foi avaliada por correlação de Pearson com os fatores do Inventário de Burnout de Maslach - versão estudantes (MBI-SS) adaptada para a língua portuguesa por Campos e Maroco (2012). A EPSS é uma medida concorrente com a ESSS, enquanto o Burnout é geralmente apresentado como uma síndrome que pode desenvolver-se na ausência de um suporte social de amigos e familiares, para além das exigências profissionais que estão na origem desta síndrome. O MBI-SS deverá assim apresentar correlação fraca e ou negativa com os fatores da ESSS.

\section{Confiabilidade}

A confiabilidade dos fatores que constituem a ESSS foi avaliada por meio da consistência interna dos fatores estimada pelo alfa de Cronbach. Considerou-se que valores $\geq 0,70$ são indicadores de confiabilidade aceitável para os fatores (Maroco \& Garcia-Marques, 2006). Foi estimada ainda a confiabilidade composta segundo proposta de Fornell e Larcker (1981) e a mesma foi considerada adequada quando igual ou superior a 0,70 .

\section{Análise Fatorial de $2^{a}$ Ordem}

Com o objetivo de compreender a importância das diferentes dimensões do suporte social no construto "Suporte Social" foi realizada uma análise fatorial confirmatória de $2^{\mathrm{a}}$ ordem onde o Suporte Social é um fator de $2^{\mathrm{a}}$ ordem que se manifesta nas dimensões de suporte social avaliadas pela ESSS.

\section{Resultados}

\section{Sensibilidade Psicométrica}

A distribuição das pontuações em cada um dos itens da ESSS é apresentada na Tabela 3.

Tabela 3

Estatísticas Descritivas dos 15 Itens da ESSS (erro-padrão assimetria=0,076; erro-padrão curtose=0,153; $n=1,023$ )

\begin{tabular}{lcccccc}
\hline Itens & Média & Mediana & Assimetria & Curtose & Mínimo & Máximo \\
\hline SS1r & 3,32 & 3 & 0,289 & $-0,753$ & 1 & 5 \\
SS2 & 4,30 & 5 & $-1,239$ & 1,590 & 1 & 5 \\
SS3 & 3,44 & 3 & $-0,534$ & 0,419 & 1 & 5 \\
SS4 & 3,80 & 4 & $-0,797$ & 0,999 & 1 & 5 \\
SS5 & 4,41 & 5 & $-1,699$ & 3,567 & 1 & 5 \\
SS6r & 3,44 & 3 & $-0,055$ & $-1,149$ & 1 & 5 \\
SS7 & 4,09 & 4 & $-1,151$ & 1,193 & 1 & 5 \\
SS8 & 4,13 & 4 & $-1,073$ & 1,300 & 1 & 5 \\
SS9r & 3,29 & 3 & $-0,039$ & $-1,285$ & 1 & 5 \\
SS10 & 4,24 & 4 & $-1,257$ & 1,900 & 1 & 5 \\
SS11 & 3,40 & 3 & $-0,598$ & 0,260 & 1 & 5 \\
SS12 & 3,78 & 4 & $-0,642$ & 0,676 & 1 & 5 \\
SS13r & 2,25 & 2 & 0,911 & 0,494 & 1 & 5 \\
SS14r & 2,50 & 2 & 0,686 & $-0,195$ & 1 & 5 \\
SS15r & 2,72 & 3 & 0,479 & $-0,676$ & 1 & 5 \\
\hline
\end{tabular}


Todos os pontos da escala foram utilizados (Min.=1, Máx.=5) e a maioria dos itens apresenta mediana e média próximas do ponto central da escala ou de pontuações que indicam valores acima da média com a satisfação do suporte social. Não se observam valores de assimetria e curtose que indiquem problemas de sensibilidade psicométrica ou desvios à distribuição normal que inviabilizem a análise das qualidades psicométricas, nomeadamente da análise fatorial confirmatória da ESSS (Kline, 2004).

\section{Validade Relacionada com o Construto}

Validade Fatorial. A análise fatorial confirmatória da estrutura de 4 fatores da ESSS na amostra global revelou um mau ajustamento à matriz de variância-covariância dos 15 itens da ESSS $\left(\chi^{2} / g l=8,966\right.$; CFI $=0,840$; GFI $=0,901$, TLI $=0,800$ RMSEA $=0,088$, IC $_{90 \%}$ RMSEA ]0,083; 0,094[). A análise dos índices de modificação produzidos pelos AMOS sugeriu que o SS1 e SS3, ambos do fator SA, são manifestações também de IN e AS. De forma semelhante, o item SS6 aparece correlacionado com o fator AS. Com o intuito de manter a validade discriminante do modelo tetra-fatorial da ESSS e melhorar a qualidade de ajustamento global, e depois de inspecionar os itens na sua relação com o conceito e construto, procedeu-se à remoção destes itens. A nova estrutura, que apresentou um ajustamento aceitável $\left(\chi^{2} / g l=4,534 ; \mathrm{CFI}=0,943\right.$; $\mathrm{GFI}=0,964, \mathrm{TLI}=0,922$;
RMSEA=0,059; IC $_{90 \%}$ RMSEA ]0,051; 0,067[), ficou constituída por 4 fatores com 3 itens cada como exposto na Figura 1.

O modelo ajustado em simultâneo na amostra portuguesa e brasileira mostrou-se adequado $\left(\chi^{2} / g l=4,181\right.$; CFI $=0,864 ;$ GFI $=0,922$, TLI $=0,859$; RMSEA $=0,056$; $\mathrm{IC}_{90 \%}$ RMSEA ]0,051; 0,061[). A análise de invariância do modelo fatorial refinado, assumindo a mesma estrutura para ambos os países (invariância configuracional) revelou a inexistência de diferenças significativas entre os pesos fatoriais dos dois países $\left[\Delta \chi^{2}(8)=10,67 ; p=0,260\right]$. Contudo, as médias (interceptos) dos itens $\left[\Delta \chi^{2}(12)=116,201\right.$; $p<0,001]$ e as correlações entre os fatores diferem significativamente entre os dois países $\left[\Delta \chi^{2}(10)=68,342 ; p<0,001\right]$. Assim, pelo fato de existir igualdade estatística apenas nos pesos fatoriais dos itens, entende-se que a ESSS apresenta apenas invariância fraca entre Portugal e o Brasil.

\section{Validade Convergente e Validade Discriminante}

O cálculo da Variância Extraída Média para cada fator revela que a validade convergente dos fatores está aquém do valor desejável: $\mathrm{VEM}_{\mathrm{SA}}=0,393 ; \mathrm{VEM}_{\mathrm{IN}}=0,479$; VE$\mathrm{M}_{\mathrm{SF}}=0,426 \mathrm{eVEM}_{\mathrm{AS}}=0,469$. A comparação dos valores de VEM com o quadrado da correlação entre fatores (ver, por exemplo, Fornell \& Larcker, 1981; Maroco 2010) indica que, na presente amostra, apenas os fatores SA e IN não apresentaram validade discriminante.

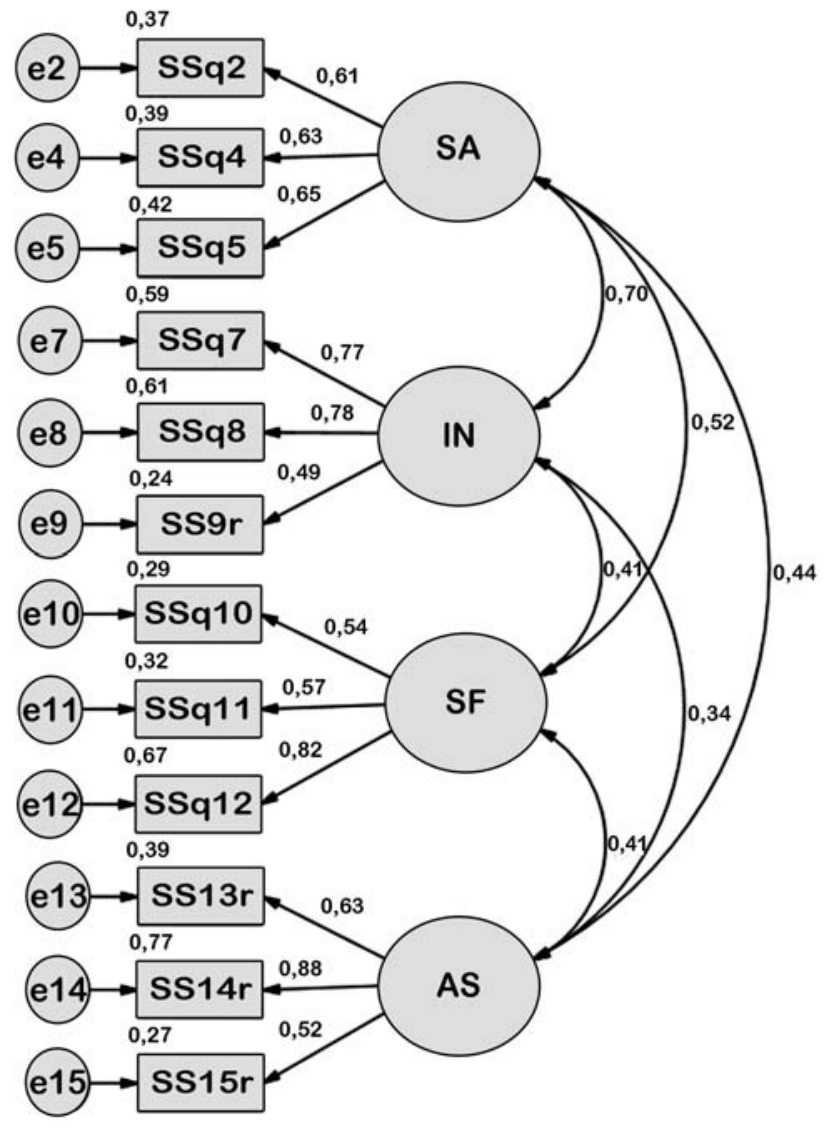

Figura 1. Pesos fatoriais e confiabilidade individual dos itens da ESSS refinada e correlações entre fatores na amostra global (Portugal e Brasil). Os itens revertidos são identificados com um "r" $\left(\chi^{2} / g l=4,534 ;\right.$ CFI $=0,943 ;$ GFI $=0,964$, TLI $=0,922$; RMSEA $=0,059$; IC90\% RMSEA ]0,051;0,067[). 
Marôco, J. P., Campos, J. A. D. B., Vinagre, M. G. \& Pais-Ribeiro, J. L. (2014). Adaptação Transcultural Brasil-Portugal da Escala de Satisfação com o Suporte Social para Estudantes do Ensino Superior.

\section{Validade de Critério}

As correlações entre os fatores da ESSS e os fatores da EPSS (um instrumento correntemente utlizado no Brasil para avaliar a perceção do Suporte Social), depois de assegurada a validade fatorial da estrutura tri-fatorial da EPSS $\left(\chi^{2} / \mathrm{gl}=4,765 ; \mathrm{CFI}=0,938 ; \mathrm{GFI}=0,901\right.$, TLI $=0,929$; RMSEA $=0,061 ; \mathrm{IC}_{90 \%}$ RMSEA ]0,057; 0,064[) revelaram correlações positivas entre os fatores da ESSS e os fatores da EPSS (Tabela 4). Relativamente à validade divergente, observam-se correlações fracas e maioritariamente negativas dos fatores da ESSS com os fatores do MBI-SS demonstrando a validade divergente da escala de satisfação com o suporte social e o Inventário de Burnout de Maslach (MBI-SS; Tabela 4).

Tabela 4

Matriz de Correlação de Pearson para os Fatores da ESSS e os Fatores da EPSS e do MBI-SS. Todas as Correlações são Significativas para $p<0,05(n=1,023)$

\begin{tabular}{lcccccc}
\hline \multirow{2}{*}{ Fatores } & \multicolumn{3}{c}{ Fatores EPSS** } & \multicolumn{3}{c}{ Fatores MBI-SS*** } \\
\cline { 2 - 7 } ESSS & SE & SI & SIF & Ex & Desc & EP \\
\hline SA & 0,437 & 0,38 & 0,357 & $-0,153$ & $-0,194$ & $-0,147$ \\
IN & 0,606 & 0,473 & 0,396 & $-0,125$ & $-0,079$ & 0,081 \\
SF & 0,342 & 0,276 & 0,295 & $-0,294$ & $-0,257$ & 0,181 \\
AS & 0,262 & 0,246 & 0,188 & $-0,3$ & $-0,194$ & 0,134 \\
\hline
\end{tabular}

Nota. *SA: Satisfação com amizades, IN: Intimidade, SF: Satisfação com a família, AS: Atividades sociais. **SE: Suporte emocional, SI: Suporte Instrumental, SIF: Suporte de Informação; ***Ex: Exaustão, Desc: Descrença, EP: Eficácia Profissional.

A Satisfação com o Suporte Social como Fator de $2^{a}$ Ordem

O suporte empírico para definir a satisfação com o suporte social como um fator hierárquico de $2^{\mathrm{a}}$ ordem - a Satisfação com o Suporte Social (ESSS) - e a forma como este se manifesta nas diferentes dimensões do suporte social, foi avaliado com a análise fatorial confirmatória de $2^{\mathrm{a}}$ ordem. $\mathrm{O}$ modelo de $2^{\mathrm{a}}$ ordem apresentou um bom ajustamento a ambos os países $\left(\chi^{2} / g l=2,820 ; \mathrm{CFI}=0,939 ; \mathrm{GFI}=0,953\right.$, $\mathrm{TLI}=0,919 ; \mathrm{RMSEA}=0,042 ; \mathrm{IC}_{90 \%}$ RMSEA $] 0,036 ; 0,048[$ ) mostrando a invariância configuracional do modelo de $2^{\mathrm{a}}$ ordem. A análise de invariância aos pesos fatoriais apontou para invariância de medida fraca $\left[\Delta \chi^{2}(8)=10,962\right.$; $p=0,204]$, mas não dos coeficientes estruturais (coeficientes das trajetórias do fator de $2^{\mathrm{a}}$ ordem para os fatores de $1^{\mathrm{a}}$ ordem) $\left[\Delta \chi^{2}(4)=11,052 ; p=0,026\right]$. Na Figura 2, pode-se notar que os coeficientes estruturais da ESSS para os fatores de $1^{\mathrm{a}}$ ordem são, de um modo geral, ligeiramente mais elevados na amostra portuguesa para os fatores SF e AS.
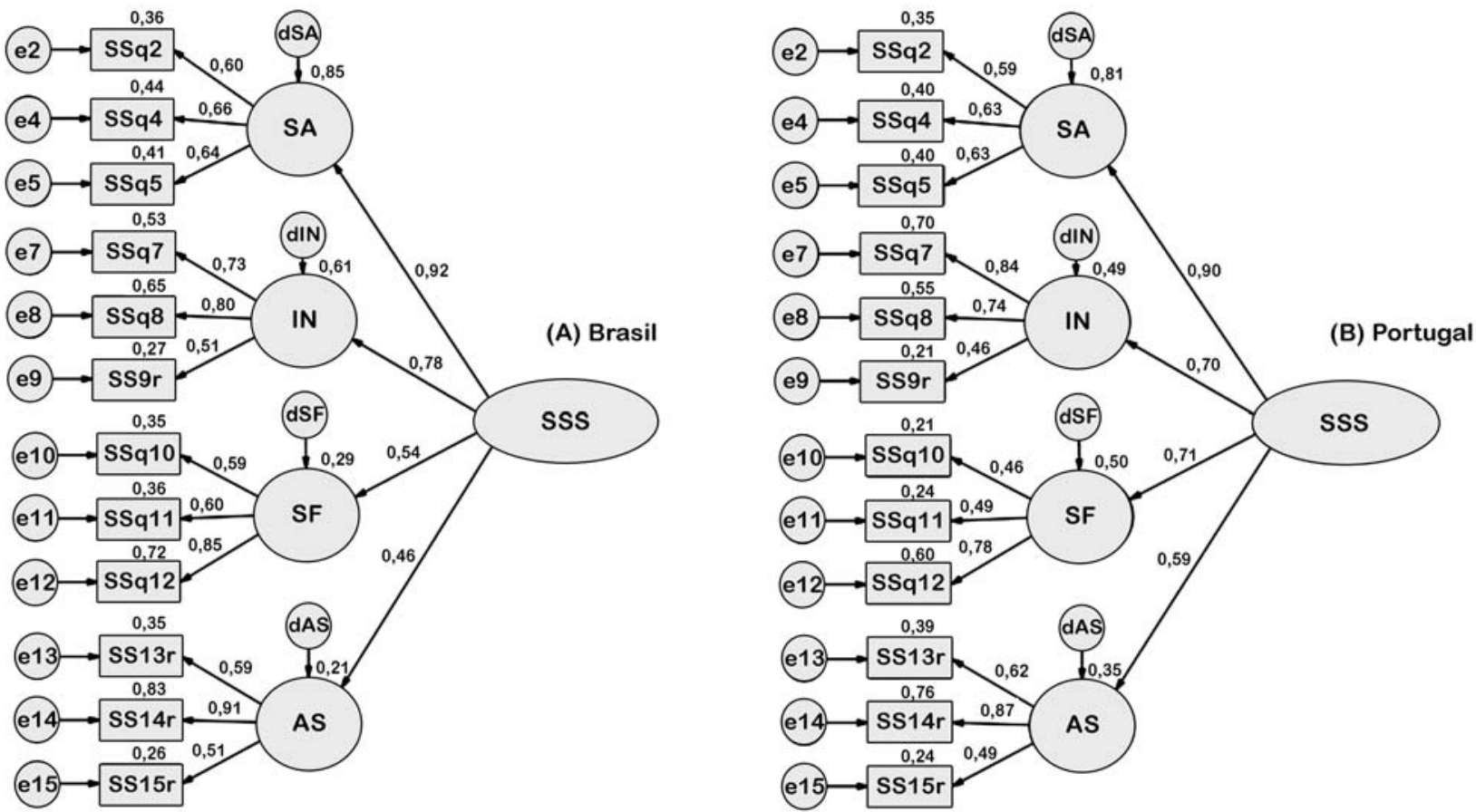

Figura 2. Modelo de $2^{\mathrm{a}}$ ordem da Satisfação com o Suporte Social (ESSS) no Brasil (A) e em Portugal (B). 
A Satisfação com o Suporte Social manifesta-se com maior intensidade na satisfação com os amigos (SA; $\left.\beta_{\mathrm{Br}}=0,92 ; \beta_{\mathrm{Pt}}=0,90\right)$ seguida da satisfação com as intimidades (IN; $\beta_{\mathrm{Br}}=0,78 ; \beta_{\mathrm{Pt}}=0,70$ ). As maiores diferenças na estrutura da satisfação com o suporte social ocorrem na dimensão suporte familiar (SF). Os estudantes portugueses atribuem maior importância ao suporte familiar $\left(\beta_{\mathrm{Pt}}=0,71\right)$ do que os estudantes brasileiros $\left(\beta_{\mathrm{Br}}=0,54\right)$. De forma semelhante, os estudantes portugueses valorizam mais as atividades sociais $\left(\mathrm{AS} ; \beta_{\mathrm{Pt}}=0,59\right)$ na satisfação com o suporte social do que os estudantes brasileiros $\left(\beta_{\mathrm{Br}}=0,46\right)$.

\section{Confiabilidade}

A análise da consistência interna dos fatores revelou valores no limite da confiabilidade adequada $\left(\alpha_{\mathrm{SA}}=0,656\right.$; $\alpha_{\mathrm{IN}}=0,673 ; \alpha_{\mathrm{SF}}=0,649 ; \alpha_{\mathrm{AS}}=0,696$; Maroco \& Garcia-Marques, 2006) que, dado se referir a três itens, os torna aceitáveis. É também de referir que o coeficiente $\alpha$ é o limite inferior da verdadeira confiabilidade e que é penalizado por um número reduzido de itens por construto. A confiabilidade composta (CC) é um melhor estimador da confiabilidade do construto. Os valores de CC indicam confiabilidade adequada para todos os fatores IN e AS $\left(\mathrm{CC}_{\mathrm{IN}}=0,72 ; \mathrm{CC}_{\mathrm{AS}}=0,72\right)$, mas ligeiramente abaixo do desejável para os fatores $\mathrm{SF}$ e $\mathrm{SA}\left(\mathrm{CC}_{\mathrm{SA}}=0,66 ; \mathrm{CC}_{\mathrm{SF}}=0,68\right.$; Fornell \& Larcker, 1981).

\section{Discussão}

O suporte social (SS) é uma variável emergente na literatura como um determinante do bem estar físico, social e psicológico (Demaray \& Malecki, 2006; Rothon et al., 2011). No contexto universitário, a importância do SS no bem-estar psicológico dos estudantes e a sua influência sobre o desempenho acadêmico e a satisfação com os estudos tem sido bem documentada (Gecková et al., 2003; O'Donovan \& Hughes, 2007; Weir \& Okun, 1989). É, assim, de relevante importância o desenvolvimento de instrumentos de medida do SS devidamente adaptados à população onde o SS deve ser medido.

Neste estudo, fizemos a adaptação transcultural Brasil-Portugal da ESSS de Pais-Ribeiro (1999), numa amostra de 1.023 estudantes do ensino superior de ambos os países. Foram utilizados os diversos procedimentos propostos na adaptação cultural de instrumentos de medida psicométrica, avaliando as facetas cognitivas dos itens e melhorando a validade relacionada com construto, validade relacionada com um critério e da confiabilidade das subescalas da ESSS. Relativamente à versão original da ESSS, foram removidos os itens que se apresentaram correlacionados com mais de um fator reduzindo a validade discriminante da escala e a qualidade do ajustamento do modelo original. A inversão dos itens pode ter dificultado a sua percepção, resultando em erros de medida mais elevados do que observado nos itens formulados na mesma direção. A remoção destes itens permitiu que a ESSS ficasse com os mesmos itens por dimensão sem comprometer a estrutura cognitiva da escala e a relação entre conceito, construto, e medida, como defende Markus (2008) e simultaneamente, observou-se melhora significativa da qualidade do ajustamento global. O modelo final apresentou VEM próximo dos valores desejáveis, mas a validade convergente das subescalas não ficou demonstrada. Uma possível solução, para melhorar a validade convergente pode passar por aumentar o número de itens em cada escala. Não se observou também validade discriminante entre os fatores SA e IN. Este fato é justificável pela VEM ser inferior a 0,50 nesses fatores e a correlação entre os mesmos ser consideravelmente forte.

Diferentes culturas utilizam de forma diferente o suporte social (Kim et al., 2008) uma vez que, as mesmas podem ser consideradas coletivistas ou individualistas o que pode influenciar, diretamente, o conceito e o construto de SS. O suporte social com os amigos (SA) e as intimidades (IN) são fatores que, em culturas de origem latina, podem apresentar fortes correlações. Por definição as culturas coletivistas salientam as relações de interdependência entre as pessoas enquanto as culturas individualistas salientam a independência pessoal dos outros (Triandis, 1994). Portugal e Brasil são culturas coletivistas (Hofstede, 1994). Este tipo cultural explica assim as relações de interdependência e a sobreposição parcial do suporte social com amigos e com as intimidades.

A análise de invariância revelou invariância configuracional e invariância de medida fraca. O modelo de medida pode ser usado nos dois países, contudo, a comparação de valores médios dos itens dos respectivos fatores entre países não é garantida uma vez que os itens apresentaram um funcionamento diferencial.

A análise de $2^{\mathrm{a}}$ ordem revela que a satisfação com o Suporte Social se manifesta com maior impacto na satisfação com os amigos entre os estudantes dos dois países. Contudo, os estudantes portugueses valorizam mais o suporte da família e das atividades sociais na satisfação com o suporte social do que os brasileiros o que pode ser justificado pelas disparidades culturais entre os países.

Diante dos resultados apresentados, entende-se que a ESSS pode ser utilizada tanto em Portugal quanto no Brasil para avaliar a satisfação com o suporte social, mas a comparação entre os resultados dos dois países não pode ser garantida. $\mathrm{O}$ fato de algumas qualidades psicométricas da ESSS observada na presente amostra poderem ser classificadas apenas como aceitáveis pode ser explicado pela heterogeneidade da amostra utilizada que inclui estudantes de diferentes áreas de estudo e de diferentes sistemas de ensino (público/privado). A presente amostra tem por isso maior variabilidade do que aquela que geralmente se observa em outros estudos de qualidades psicométricas onde as amostras são de menor dimensão e mais homogêneas relativamente a características sócio-educacionais. Assim, a heterogeneidade observada, que condiciona as qualidades psicométricas, apresenta-se não como uma limitação, mas sim como uma vantagem comparativa relativamente a outros es- 
Marôco, J. P., Campos, J. A. D. B., Vinagre, M. G. \& Pais-Ribeiro, J. L. (2014). Adaptação Transcultural Brasil-Portugal da Escala de Satisfação com o Suporte Social para Estudantes do Ensino Superior.

tudos com amostras mais homogêneas e eventualmente com melhores propriedades psicométricas. Estes dados reforçam a utilidade da ESSS para avaliar a satisfação com o suporte social em estudantes do ensino superior de diferentes áreas de estudo e sistemas de ensino.

Em conclusão, a Escala de Satisfação com o Suporte Social (ESSS) adaptada transculturalmente para o português, quando aplicada a estudantes universitários brasileiros e portugueses, é um instrumento que produz dados confiáveis e válidos tanto no contexto português quanto brasileiro. A ESSS revelou invariância dos pesos fatoriais dos itens entre os dois países possibilitando assim a utilização de uma versão comum. A escala ESSS pode, assim, ser utilizada com validade e confiabilidade na avaliação da satisfação com o suporte social quer em ambiente de investigação científica, quer na prática profissional de psicólogos e educadores que dão apoio a estudantes do ensino superior e à resolução de seus problemas acadêmicos, de orientação profissional, bem como de saúde física e mental.

\section{Referências}

Baptista, M. N., Baptista, A. S., \& Torres, E. R. (2006). Associação entre suporte social, depressão e ansiedade em gestantes. PSIC - Revista de Psicologia da Vetor Editora, 7, 39-48.

Berkman, L. F., \& Glass, T. (2000). Social integration, social networks, social support \& health. In L. Berkman \& I. Kawachi (Eds.), Social epidemiology (pp. 137-173). New York: Oxford University Press.

Bradley, C. (1994). Translation of questionnaires for use in different languages and cultures. In C. Bradley (Ed.), Handbook of psychology and diabetes (pp. 43-55). Chur, Switzerland: Hardwood Academic Press.

Callaghan, P., \& Morrissey, J. (1993). Social support and health: A review. Journal of Advanced Nursing, 18, 203-210. doi:10.1046/j.1365-2648.1993.18020203.x

Campos, J. A. D. B., \& Maroco, J. (2012). Maslach Burnout Inventory - Student Survey: Portugal-Brazil cross-cultural adaptation. Revista de Saúde Pública 5. Manuscript in preparation.

Campos, J. A. D. B., Zucoloto, M. L., Bonafé, F. S. S., Jordani, P. C., \& Maroco, J. (2011). Reliability and validity of self reported burnout in college students: A cross randomized comparison of pencil-and-paper vs. online administration. Computers in Human Behavior, 27, 1875-1883. doi:10.1016/j. chb.2011.04.011

Cobb, S. (1976). Social support as a moderator of life stress. Psychosomatic Medicine, 38, 300-314.

Chor, D., Griep, R. H., Lopes, C. S., \& Faerstein, E. (2001). Medidas de rede e apoio social no Estudo Pró-Saúde: Prétestes e estudo piloto. Cadernos de Saude Publica, 17, 887-896.

Coelho, M., \& Pais-Ribeiro, J. (2000). Influência do suporte social e do coping sobre a percepção subjectiva de bem-estar em mulheres submetidas a cirurgia cardíaca. Psicologia, Saúde \& Doenças, 79-87.

Cohen, S., \& Lemay, E. P. (2007). Why would social networks be linked to affect and health practices? Health Psychology, 26, 410-417.

Cramer, D., Henderson, S., \& Scott, R. (1997). Mental health and desired social support: A four-wave panel study. Journal of Social and Personal Relationships, 14, 761-775. doi:10.1177/0265407597146003
De Raad, B. (1998). Five big, big five issues: Rationale, content, structure, status, and crosscultural assessment. European Psychologist, 3, 113-124. doi:10.1037/1016-9040.3.2.113

Demaray, M., \& Malecki, C. (2006). A review of the use of social support in anti-bullying programs. Journal of School Violence, 5(3), 51-70. doi:10.1300/J202v05n03 05

Diong, S. M., Bishop, G. D., Enkelmann, H. C., Tong, E. M. W., Why, Y. P., Ang, J. C. H., \& Khader, M. (2005). Anger, stress, coping, social support and health: Modelling the relationships. Psychology \& Health, 20, 467-495. doi:10.1080/088704404 0512331333960

Ferreira, T., Pais-Ribeiro, J., \& Guerreiro, M. (2004). Estudo de adaptação da Escala de Satisfação com o Suporte Social em doentes submetidos a revascularização do miocárdio. Revista Referência, 11, 5-16.

Fornell, C., \& Larcker, D. F. (1981). Evaluating structural equation models with unobservable variables and measurement error. Journl of Marketing Research, 18, 39-50.

Gecková, A., van Dijk, J., Stewart, R., Groothoff, J., \& Post, D. (2003). Influence of social support on health among gender and socio-economic groups of adolescents. European Journal of Public Health, 13, 44-50. doi:10.1093/eurpub/13.1.44

Gonçalves, T. R., Pawlowski, J., Bandeira, D. R., \& Piccinini, C. A. (2011). Avaliação de apoio social em estudos brasileiros: Aspectos conceituais e instrumentos. Ciência \& Saúde Coletiva, 16, 1755-1769.

Gwaltney, C. J., Shields, A. L., \& Shiffman, S. (2008). Equivalence of electronic and paper-and-pencil administration of patient-reported outcome measures: A meta-analytic review. Value in Health, 11, 322-333. doi:10.1111/j.15244733.2007.00231.x

He, J., \& Van de Vijver, F. (2012). Bias and equivalence in crosscultural research. Online Readings in Psychology and Culture. Retrieved from http://scholarworks.gvsu.edu/orpc/vol2/iss2/8

Herdman, M., Fox-Rushby, J., \& Badia, X. (1998). A model of equivalence in the cultural adaptation of HRQoL instruments: The universalist approach. Quality of Life Research, 7, 323-335.

Hofstede, G. (1994). Cultures and organizations: Intercultural cooperation and its importance for survival. London: HarperCollins.

Kim, H. S., Sherman, D. K., \& Taylor, S. E. (2008). Culture and social support. American Psychologist, 63, 518-526. doi:10.1037/0003-066X

Kline, R. B. (2004). Principles and practice of structural equation modeling ( $2^{\text {nd }}$ ed.). New York: The Guilford Press.

Markus, K. (2008). Constructs, concepts and the worlds of possibility: Connecting the measurement, manipulation, and meaning of variables. Measurement, 62, 54-77. doi: $10.1080 / 15366360802035513$ Retrieved from http:// www.tandfonline.com/doi/pdf/10.1080/15366360802035513

Maroco, J. (2010). Análise de equações estruturais. Lisboa, Portugal: ReportNumber.

Maroco, J., \& Garcia-Marques, T. (2006). Qual a fiabilidade do alfa de Cronbach? Questões antigas e soluções modernas? Laboratório de Psicologia, 4(1), 65-90.

O'Donovan, A., \& Hughes, B. (2007). Social support and loneliness in college students: Effects on pulse pressure reactivity to acute stress. International Journal of Adolescent Medicine and Health, 19, 523-528.

Pais-Ribeiro, J. L. (1999). Escala de Satisfação com o Suporte Social (ESSS). Análise Psicológica, 17, 547-558. 
Pais-Ribeiro, J. L., \& Guterres, C. (2001). Social support and quality of life in chronic mental disease patients submitted to different housing treatment programs. Quality of Life Research, 10, 275-275.

Pais-Ribeiro, J. L., \& Ponte, A. C. (2009). Propriedades métricas da versão portuguesa da Escala de Suporte Social do MOS (MOS Social Support Survey) com idosos. Psicologia, Saúde \& Doenças, 10, 163-174.

Rothon, C., Head, J., Klineberg, E., \& Stansfeld, S. (2011). Can social support protect bullied adolescents from adverse outcomes? A prospective study on the effects of bullying on the educational achievement and mental health of adolescents at secondary schools in East London. Journal of Adolescence, 34, 579-588. doi:10.1016/j.adolescence.2010.02.007

Santos, C., Pais-Ribeiro, J., \& Lopes, C. (2003). Estudo de adaptação da Escala de Satisfação com o Suporte Social (ESSS) a pessoas com diagnóstico de doença oncológica. Psicologia, Saúde \& Doenças, 4, 185-204.

Sarason, I. G., Levine, H. M., Basham, R. B., \& Sarason, B. R. (1983). Assessing social support: The Social Support Questionnaire. Journal of Personality and Social Psychology, 44, 127-139.

Sherbourne, C. D. (1992). Social functioning: Social activity limitations measure. In A. Stewart \& J. Ware (Eds.), Measuring functioning and well-being: The Medical Outcomes Study Approach (pp. 173-181). Durham, UK: Duke University Press.

Sherbourne, C. D., \& Stewart, A. L. (1991). The MOS Social Support Survey. Social Science \& Medicine, 32, 705-714.

Silva, I., Pais Ribeiro, J., Cardoso, H., \& Ramos, H. (2003). Efeitos do apoio social na qualidade de vida, controlo metabólico e desenvolvimento de complicações crónicas em indivíduos com diabetes. Psicologia, Saúde \& Doenças, 4, 21-32.

Singer, J. E., \& Lord, D. (1984). The role of social support in coping with chronic or life-threatning illness. In A. Baum, S. Taylor, \& J. Singer (Eds.), Handbook of psychology and health (Vol. 4, pp. 269-278). Hillsdale, NJ: Laurence Erlbaum.

Siqueira, M. M. M. (2008). Construção e validação da Escala de Percepção de Suporte Social. Psicologia em Estudo, 13, 381-388.

Taylor, S. E. (2008). Fostering a supportive environment at work. The Psychologist-Manager Journal, 11, 265-283. doi:10.1080/10887150802371823

Taylor, S. E., Seeman, T. E., Eisenberger, N. I., Kozanian, T. A., Moore, A. N., \& Moons, W. G. (2010). Effects of a supportive or an unsupportive audience on biological and psychological responses to stress. Journal of Personality and Social Psychology, 98, 47-56. doi:10.1037/a0016563

Triandis, H. C. (1994). Theoretical and methodological approaches to the study of collectivism and individualism. In U. Kim, H. C. Triandis, C. Kagitcibasi, S. C. Choi, \& G. Yoon (Eds.), Individualism and collectivism: Theory, methods, and applications (pp. 41-51). Thousand Oaks, CA: Sage.

Uchino, B. N. (2006). Social support and health: A review of physiological processes potentially underlying links to disease outcomes. Journal of Behavioral Medicine, 29, 377-387. doi:10.1007/s10865-006-9056-5

Weir, R. M., \& Okun, M. A. (1989). Social support, positive college events, and college satisfaction: Evidence for boosting effects. Journal of Applied Social Psychology, 19, 758-771. doi:10.1111/j.1559-1816.1989.tb01257.x 